\title{
Quality of Life in Patients with Chronic Oral Mucosal Conditions: A Qualitative Research
}

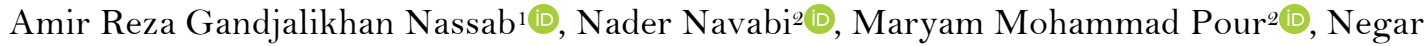 \\ Charrosta $^{3}\left(\mathbb{0}\right.$, Maryam Alsadat Hashemipour ${ }^{3}[0$
}

'School of Medicine, Kerman University of Medical Sciences, Kerman, Iran.

${ }^{2}$ Department of Oral Medicine, Dental School, Kerman University of Medical Science, Kerman, Iran.

${ }^{3}$ Dental School, Kerman University of Medical Science, Kerman, Iran.

Correspondence: Maryam Alsadat Hashemipour, Kerman dental School, Shafa Street, Kerman, Iran. E-mail: m_s_hashemipour@yahoo.com

Academic Editor: Alessandro Leite Cavalcanti

Received: 15 May / Review: 05 July 2020 / Accepted: 10 August 2020

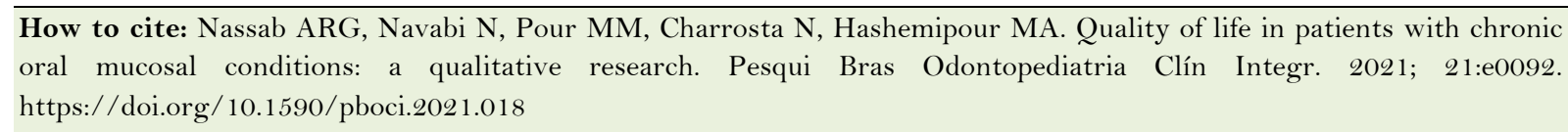

\begin{abstract}
Objective: To use qualitative research methods to evaluate the experiences of patients with chronic oral diseases. Material and Methods: Purposive sampling was used to recruit patients from the Oral Medicine Unit of Kerman University Dental School. An experienced independent facilitator convened the focus groups and conducted individual interviews in a non-clinical setting. Focus groups were mixed regarding their gender, age, chronic oral mucosal condition, time since diagnosis, and severity. A total of 39 patients participated in the study, including patients with oral lichen planus, pemphigus, recurrent aphthous stomatitis and leukoplakia. Results: Analysis of the interviews revealed that patient views could be divided into the following themes: biopsychosocial issues, treatment limitations and side effects, unpredictability of the conditions and the potential for malignant transformation. Chronic oral mucosal conditions affected patients' daily lives in various areas, from physical health and functioning to concerns about their future. Conclusion: The oral medicine practitioner's role in treating patients with chronic oral mucosal diseases extended beyond active management and symptomatic relief to the management of all aspects of these conditions that affected their daily lives.
\end{abstract}

Keywords: Quality of Life; Oral Medicine; Lichen Planus; Stomatitis, Aphthous. 


\section{Introduction}

The oral mucosa's chronic diseases include autoimmune and inflammatory diseases, oral soft tissue diseases, oral sores, and lichenoid lesions. Like other oral diseases, these conditions can lead to significant physical effects and social and psychological consequences for patients [1]. Some of these diseases lead to lifethreatening conditions and the chronic conditions of the oral mucosa may affect the patient's everyday life, necessitating long and expensive treatments. In addition, the side effects of treatment can also affect the patient's daily life [Q].

Oral and dental disease specialist's role in treating patients with chronic diseases of the oral mucosa is beyond the active management or symptomatic relief and includes managing the patients and the diseases that affect all the aspects of daily life [3]. Assessment of patients' perception of their health's impact on their daily lives has long been a matter of debate in the medical community [4].

In 2001, the Department of Health in England evaluated the critical role of patients with chronic diseases in health care and patients' positive attitudes to refer to health care professionals. Based on that and interviews with patients, it was concluded that patients had a better understanding of their disease than their doctors or nurses and better understood the treatment process and responded well to the treatment [5]. Today, patients with chronic oral diseases are the recipients of care and can be converted to key decisionmakers in the treatment process. In addition, the role of patients in providing essential information on living with chronic conditions has been demonstrated.

Qualitative research methods whereby patients are interviewed according to their conditions allow them to see their disease through their perspectives [6,7]. Stainer et al. reported that doctors could give a professional and specialized opinion through observing the patient's clinical complaints; however, the patient is the only one that can report this situation through the mental aspects and its impact on his life. Over the last few years, the use of qualitative research to collect data through in-depth interviews and focus groups has expanded. This type of research all owes extensive review of the issues and details; it also allows the interviewed person to easily express their opinion and come to group discussions [8].

Therefore, it appears that this type of data can typically bead along with medical or epidemiological data and results and enhance patient care. Reports on the evaluation of the treatment of oral diseases are limited [9-12]. Riordain et al. conducted the only study on the impact of chronic oral diseases on daily life, in which the analysis of interviews revealed that patient perspectives revealed bio-psycho-social issues, limitations and side effects of treatment, unpredictable conditions, the possibility of malignant lesions and issues related to the health care [6].

The purpose of this study was to use qualitative research methods to evaluate the experiences of patients with chronic oral diseases about their disease and to identify factors affecting their daily life in patients referred to Oral and Dental Disease Department of Kerman Dental School.

\section{Material and Methods}

Study Design

In this qualitative study, interviewing groups were selected from patients referred to the Department of Oral and Dental Diseases of Kerman Dental School, who had chronic oral diseases such as aphthous stomatitis, pemphigus, lichen planus, and leukoplakia. Information was collected through interviews and group discussions (Focus Group). People with different types of chronic oral diseases were placed in 6 separate groups. In this study, the diagnosis was made for the patients who had leukoplakia based on biopsy and clinical 
appearance. Besides, patients with dysplasia (mild, moderate, and severe dysplasia) were not included in the study.

The patients were approached by a person who was not aware of the aim of this study. Then, the purpose of this research was explained to the patients before the interview and those tending participation in this study entered the research. Therefore, there was not any selection biases in this study. In other words, the patients in each group explained their diseases and at the end of the interview, they were asked to recall the name of their diseases. The interview with patients was carried out by researchers who were not aware of the disease of the patients.

The gender of the subjects in the study had no effects on the audience`s feedback; however, an attempt was made to include patients of both genders in each group. Also, the education level was not considered in choosing the participants, and they had weak to moderate economic ability. The compositions of these groups were selected so that in each group, some patient sex presence less illness and some patients had a long history of their disease. For the interview, the patients were asked to come to the Dental School with a prior arrangement. Two researchers (two dental students) participated in all the interviews (as interviewer and observer), who were not aware of the patients' disease.

The first person was responsible forth interview and the second was in charge of recording. Before starting the interviews, the two researchers were coordinated. An independent expert (an oral and dental disease specialist) who was aware of the issues related to oral chronic mucosal diseases gathered all the groups in a non-clinical environment and interviews were conducted. The sampling was carried out purposefully, and the focus groups were different in terms of gender, age, diagnosis and disease severity. At least 35 minutes were spent on each interview.

During the interviews, the patients were asked about the development of the disease and the impact of the disease on daily life, social relations, career and family relationships. In addition, the patients were asked about their opinion about their disease. The interview consisted of in-depth, open and semi-structured questions. At the beginning of each interview, general questions and then specific questions (to avoid a general discussion) were asked. Attempts were made to make the questions simple and without prejudice to the audience.

At first, the interviewer asked questions such as "Can you explain further?" and "Can you give an example to us?". At the end of each section of the interview, the negotiations' outcome was concluded and summarized for other members of the groups to ensure the correctness of the views. Two participants were reluctant to participate in the group study because of stress; they were interviewed individually later. None of the participants wrote their names, and a number was assigned to each participant. Interviews were written on the paper by two dental students after recording and the comments of the participants were coded individually by the specialist, which continued until data saturation. Finally, data were evaluated according to the content analysis; the analyzed data were classified, and the number of respondents for each category was determined. In addition, to increase the scientific accuracy and validation, codes from each interview were given to each participant to ensure that researchers collected the patients' views.

In this study, the following items were considered to increase the reliability and ineffectiveness of patients' conversations with each other: 1) Patients were unaware of each other's diseases; 2) Before coding and final examination, they were contacted to confirm their words and in case of inconsistency, the patient's sentences were deleted. Therefore, the likelihood of affecting other patients was minimized; 3) The initial 
dossier of the patients referred to the ward was available. If the patient mentioned that there were no complaints in the initial visit, questions would be asked to confirm it.

Ethical Aspects

The study was confirmed by the Ethics Committee of Kerman University of Medical Sciences (Protocol No. IR.KMU.REC.920109). Before the interview, oral consent was obtained from the patients.

\section{Results}

The in-depth interviews were performed with 39 patients (12 patients with oral aphthous ulcers, seven patients with pemphigus, 13 patients with lichen planus, and seven patients with leukoplakia) (Table1).

Table1. Demographic information of participants in the study with their chronic oral conditions.

\begin{tabular}{|c|c|c|c|c|c|}
\hline No. & Gender & Age (Years) & Education & Occupation & Type of Disease \\
\hline 1 & Female & 57 & Bachelor Degree & Retired Teacher & Lichen Planus \\
\hline 2 & Male & 27 & Diploma & Self Employee & Lichen Planus \\
\hline 3 & Female & 46 & Diploma & House Keeper & Lichen Planus \\
\hline 4 & Male & 45 & Bachelor Degree & Teacher & Lichen Planus \\
\hline 5 & Female & 43 & Diploma & Self Employee & Lichen Planus \\
\hline 6 & Male & 42 & Diploma & Self Employee & Lichen Planus \\
\hline 7 & Female & 39 & Diploma & House Keeper & Lichen Planus \\
\hline 8 & Female & 55 & Bachelor Degree & House Keeper & Lichen Planus \\
\hline 9 & Female & 41 & Diploma & House Keeper & Lichen Planus \\
\hline 10 & Male & 45 & Master & Employee & Lichen Planus \\
\hline 11 & Male & 45 & Diploma & Self Employee & Lichen Planus \\
\hline 12 & Female & 47 & Diploma & House Keeper & Lichen Planus \\
\hline 13 & Female & 52 & Diploma & House Keeper & Lichen Planus \\
\hline 14 & Female & 48 & Associate Degree & House Keeper & Pemphigus Vulgaris \\
\hline 15 & Female & 28 & Student & Student & Pemphigus Vulgaris \\
\hline 16 & Female & 42 & Diploma & Self Employee & Pemphigus Vulgaris \\
\hline 17 & Male & 41 & Associate Degree & Teacher & Pemphigus Vulgaris \\
\hline 18 & Male & 39 & Diploma & Self Employee & Pemphigus Vulgaris \\
\hline 19 & Male & 38 & Diploma & Self Employee & Pemphigus Vulgaris \\
\hline 20 & Male & 36 & Diploma & Self Employee & Pemphigus Vulgaris \\
\hline 21 & Female & 17 & Student & Student & Aphthous Stomatitis \\
\hline 22 & Female & 19 & Student & Student & Aphthous Stomatitis \\
\hline 23 & Female & 20 & Student & Student & Aphthous Stomatitis \\
\hline 24 & Female & 22 & Student & Student & Aphthous Stomatitis \\
\hline 25 & Male & 18 & Student & Student & Aphthous Stomatitis \\
\hline 26 & Male & 17 & Student & Student & Aphthous Stomatitis \\
\hline 27 & Female & 23 & Student & Student & Aphthous Stomatitis \\
\hline 28 & Female & 25 & Student & Student & Aphthous Stomatitis \\
\hline 29 & Female & 19 & Student & Student & Aphthous Stomatitis \\
\hline 30 & Female & 18 & Diploma & House Keeper & Aphthous Stomatitis \\
\hline 31 & Female & 18 & Diploma & House Keeper & Aphthous Stomatitis \\
\hline 32 & Male & 21 & Diploma & No Jobs & Aphthous Stomatitis \\
\hline 33 & Female & 58 & $<$ Diploma & House Keeper & Leukoplakia \\
\hline 34 & Female & 60 & Diploma & House Keeper & Leukoplakia \\
\hline 35 & Male & 61 & $<$ Diploma & Retired & Leukoplakia \\
\hline 36 & Male & 53 & Diploma & Self Employee & Leukoplakia \\
\hline 37 & Male & 52 & Diploma & Self Employee & Leukoplakia \\
\hline 38 & Male & 64 & Under Graduate & Retired & Leukoplakia \\
\hline 39 & Male & 61 & Under Graduate & Retired & Leukoplakia \\
\hline
\end{tabular}


The patients were assigned to various classes (with different ages, educational levels and occupations). Data were coded and then the codes were categorized. The codes for patients with the number of patients responding to each code have been listed in Table 2 .

Table 2. The codes for patients with the number of patients responding to each code.

\begin{tabular}{lc}
\hline \multicolumn{1}{c}{ Code } & N \\
\hline Change in Life Style & 18 \\
Delay in Diagnosis & 12 \\
Being Concerned that the Disease will Exacerbate & 22 \\
Receiving Different Recommendations in the Field of Disease & 15 \\
Being Seen by Several Doctors & 18 \\
Satisfaction or Dissatisfaction with Medications & 21 \\
Restrictions in Eating Spicy Food and Drinking Sour Drinks & 22 \\
Restrictions in Cool or Warm Foods and Drinks & 23 \\
Restrictions in Oral Hygiene (Brushing, Flossing and Use of Mouthwash) & 9 \\
Feelings of Inability and Depression & 12 \\
Feelings of Anxiety & 32 \\
Fear of Cancer & 21 \\
\hline
\end{tabular}

Almost all the patients wished to have more information about their conditions. For better understanding the issues and the effect of oral diseases on quality of life, major themes were categorized into 5 key issues, including the following: 1) Psychological and social issues; 2) Family problems; 3) Limitations of treatment and its side effects; 4) Unpredictability of the conditions and the potential for malignant transformation, and 5) Issues related to the diagnosis.

Psychological and Social Issues

The mouth is considered one of the most important organs of the body. Chronic mucosal diseases have a great effect on physical activity and affect psychological and social activities. In this study, 22 patients complained about burning sensation with the intake of spicy foods, sharp and sour drinks and one patient complained of a sore tongue and it's splitting, especially when he got nervous. Except for two patients, all the patients stated that this situation had caused a lot of stress in them and 12 cases, it resulted in a feeling of depression in them. Many interviews with people in this field are as follows.

\section{Family Problems}

Family problems and fear of oral transmission are some of the most important issues. In this study, eight patients complained regarding others' behavior; 12 patients stated that they did not say anything about their illness to keep it a secret. Five patients with lichen planus and leukoplakia (which might be precancerous lesions) stated that they did not say anything about it to their family.

\section{Limitations of Treatment and Its Side Effects}

Undoubtedly, for treating all the diseases mentioned above, drugs have been administered, which can sometimes cause side effects or exacerbate the patient's condition. In addition, some patients were treated by general practitioners (14 cases), and some used traditional medicines such as pomegranates and baking soda (8 cases). Five patients were not satisfied with expensive Adcortyl medicines and their paucity on the market. Some patients had experienced a period of trial and error since the early diagnosis of their disease and these 
had aggravated their condition. A number of participants also had concerns about the possible side effects of some medicines.

Unpredictability of the Conditions and the Potential for Malignant Transformation

Nearly all the participants insisted on this topic. The unpredictable nature of their illness caused some disorders and anxiety during their everyday life. Fear of cancer, the risk of transmission of disease and uncontrolled worsening of the disease were the main topics in this field.

\section{Issues Related to Diagnosis}

A total of 18 participants stated that their disease was diagnosed late. In the case of patients with leukoplakia, two patients complained that they had their disease since two years ago, but their dentist did not notice it. Table 3 has compared the results of the present study with the study of Riordain et al. [6].

Table 3. Comparison of the present study results with the study of Riordain et al. [6].

\begin{tabular}{|c|c|c|}
\hline Variables & Riordain et al. [6] & Present Study \\
\hline Number of Patients & 24 & 39 \\
\hline \multicolumn{3}{|l|}{ Sex } \\
\hline Male & 8 & 17 \\
\hline Female & 16 & 22 \\
\hline Age Range (Years) & $33-76$ & $17-64$ \\
\hline \multirow[t]{6}{*}{ Chronic Oral Diseases } & $\mathrm{N}(\%)$ & $\mathrm{N}(\%)$ \\
\hline & Oral Lichen Planus (17 / 70.9) & Oral Lichen Planus (13 / 33.2) \\
\hline & Mucous Membrane Pemphigoid (2 / 8.3) & Leokoplakia (7 / 18.0) \\
\hline & Pemphigus Vulgaris (2 / 8.3) & Pemphigus Vulgaris (7 / 18.0) \\
\hline & Recurrent Aphthous Ulceration (1/ 4.2) & Recurrent Aphthous Ulceration (12 / 30.7) \\
\hline & Orofacial Granulomatosis (2 / 8.3) & \\
\hline Number of Focus Groups & Five & Six \\
\hline Patient Complaints & $\begin{array}{l}\text { Difficulties with the Textures and } \\
\text { Temperatures of Food; Limitations on } \\
\text { Diet; Discomfort in Social Settings; High } \\
\text { Levels Stress; Depression; Side Effects of } \\
\text { Some Medications; Potential for } \\
\text { Malignant Transformation and Delay in } \\
\text { Diagnosing. }\end{array}$ & $\begin{array}{l}\text { Burning Sensation with the Intake of Spicy } \\
\text { Foods, Sharp and Sour Drinks; Stress; } \\
\text { Depression; Fear of Cancer; The Risk of } \\
\text { Transmission of Disease and Uncontrolled } \\
\text { Worsening of the Disease; Not to Say } \\
\text { Anything About it to Their Family; Side } \\
\text { Effects of Some Medicines; The Unpredictable } \\
\text { Nature of Their Illness; Delay in Diagnosing }\end{array}$ \\
\hline
\end{tabular}

\section{Discussion}

Oral mucosal diseases include autoimmune and inflammatory chronic diseases of oral soft tissues. Like other oral diseases, these diseases can cause significant physical, social and mental disabilities inpatients [1]. These patients are usually treated at oral medicine clinics and often have recurrent, painful and long-lasting disorders [13]. Chronic conditions of oral mucosa not only can affect the daily lives of patients but also a variety of treatments and their side effects can affect their daily lives [14]. Patients' personal feelings about their health affect their life quality [4]. In 2001, a specialist in America proposed a plan that described the patients' vital role in the care of their disease and experts' insight into patients with chronic diseases. These documents emphasized the value of the patient's cooperation in preparing key information related to living with chronic conditions [5].

Qualitative research methods, by which patients are interviewed about their disease, allow patients to see these diseases with their eyes. This information can add to epidemiological data that are normally recorded to enhance patient care [7]. 
A qualitative research method has been used extensively in health care in recent years. Qualitative information is gathered through interviews and focus groups, which allows a more healthy and comfortable environment and research in detail. Focus groups are interview groups that rely on regular communication with participants to achieve useful information, especially about the conditions, treatment, feelings and experiences of people [8].

Few studies have focused on the quality of life in patients with oral diseases [14,15]. These studies have been limited to patients with lichen planus [10], pemphigus [11], and oral aphthous ulcers [12]. In all these studies, the research was based on questionnaires without any interviews with patients. Some qualitative studies on dental services' fields include the quality assessment in the elderly population in America [16], a qualitative assessment of people's expectations of dentistry [17], people's expectations of emergency dental treatment in Australia [18] and evaluation of dental services (in physicians' offices) and the patients' expectations of these services in the UK [19].

The present study was performed on the impact of chronic oral diseases on the quality of life. It was the first qualitative study in Iran and the world's second study after a study by Riordain et al. [6] on chronic oral diseases.

This study showed that pain and discomfort are the main experience of patients in their daily lives, affecting some aspects of patients' lifestyles, such as changes in diet and restrictions in consuming certain beverages and foods, which was consistent with previous findings [6,15,20]. Oral pain and functional limitations can interfere with social programs, causing embarrassment to patients.

The unpredictable nature of the disease leads to frustration, doubt, sand uncertainties about the future of the patient's life. Patients with lichen planus and leukoplakia were concerned about the disease's malignant potential and other patients had concerns about the progress of their disease.

The diagnostic delay experienced by patients was another sobering issue in primary care. Patients reported significant delays in patient referral by general practitioners and dentists. In this context, the use of antibiotics and antifungal to treat the disease was significant. Studies have shown that patients with oral lesions were faced with many problems, with delayed diagnosis and treatment being the most important ones. Jovanovich et al. conducted a study in 1992 in the Netherlands on the referral pattern of patients with oral lesions and showed that family physicians normally referred patients to medical experts of various fields rather than dentists [21]. However, in most medical courses, oral diseases and their symptoms and signs weren't taught clearly as a separate chapter.

Previous authors showed that the average time wasted from initial symptoms' appearance until the patient's evaluation by a specialist in oral medicine was about 15 months [22]. Oral medicine is one of the specialties of dentistry that deals with treating mucosal lesions of the oral cavity. These lesions may be one of the oral mucosa's focal lesions, mucosal skin disease, or manifestations of different systemic diseases. Unfortunately, not many physicians and dentists are familiar with the range of capabilities, performance and features of dental and oral disease specialties. In addition to the lack of training on oral diseases for medical students and the lack of precise knowledge of experts in various fields of medicine on these diseases, many patients with oral lesions have been misdiagnosed and have received unnecessary or inappropriate treatments [23].

Low awareness of the clinical features of chronic diseases is an important concern of the patients. Previous studies have reported low knowledge levels of physicians in primary care regarding oral diseases and suggestions have been made to correct this issue $[9,24,25]$. A possible reason for the delay in diagnosis is the 
lack of regular contact between medical doctors and dentists with oral medicine specialists, according to which inappropriate referrals are made. Approximately one-third of patients in this study had been visited by a physician or dentist before referring to the Department of Oral Medicine, similar to previous studies [6,26]. Haberland et al. showed that a general practitioner had examined an average of 2.2 patients before having a visit by oral disease specialists [27]. Undoubtedly, advances in oral medicine and soft tissue diseases can result in faster and more accurate referrals [28].

Appropriate referrals decrease delays in diagnosis and result in greater will ingress to provide sufficient services. Spending unnecessary time, especially during examination sessions, can increase waiting time for patients who need to receive medical care. Increases in costs, both financially and poor management of time for consultation, can result in inadequate and inappropriate care of patients. Proper knowledge of the medical community, especially in the field of oral medicine and the performance and capabilities of experts in this field, is very important to improve the management of chronic oral diseases [6]. Research has shown that chronic oral diseases can affect different aspects of the patient's life, such as physical health, performance and concerns about the future. Therefore, it seems that improving knowledge about the oral cavity should be considered to help understand the impact of the disease on patients' lives.

It is recommended that studies be performed with more patients. Moreover, other extensive studies should be conducted in dental centers. Also, since the patients in this study were afraid of cancer or transmission, it is better for these patients to have psychiatric counseling to reduce their stress and anxiety in this regard. Furthermore, studies could be carried out by forming groups of patients and family members to better understand the patient's condition by those around them and reduce patients' anxiety.

\section{Conclusion}

Chronic diseases of the oral mucosa affect patients' daily life experience, including physical health and activity, and what is relevant to their future. The role of oral medicine practitioners in treating patients with chronic oral mucosal diseases extends beyond the active management and symptomatic relief to the management of all the aspects of these conditions that affect their daily lives.

\section{Authors' Contributions}

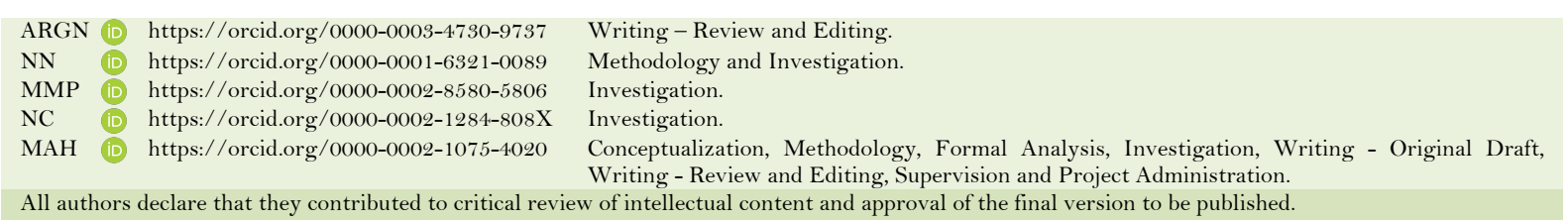

\section{Financial Support}

This study was supported by Kerman University of Medical Sciences (Grant No. 920109).

\section{Conflict of Interest}

The authors declare no conflicts of interest.

\section{Data Availability}

The data used to support the findings of this study can be made available upon request to the corresponding author.

\section{Acknowledgments}

The authors would like to thank the Research Deputy for their financial support. 


\section{References}

[1] McGrath C, Bedi R. A review of the influences of oral health on the quality of life. Int J Health Prom Educ 1999; 37(4):116-9. https://doi.org/10.1080/14635240.1999.10806111

[2] Hashemipour MA, Shoryabi A, Adhami S, Mehrabizadeh Honarmand H. Extensive focal epithelial hyperplasia. Arch Iran Med 2010;13(1):48-52.

[3] Riordain RN, McCreary C. The use of quality of life measures in oral medicine: a review of the literature. Oral Dis 2010; 16(5):419-30. https://doi.org/10.1111/j.1601-0825.2009.01647.x

[4] Bowling A. Measuring Disease: A review of Disease Specific Quality of Life Measurement Scales. 2nd. ed. Ballmoor: Open University Press; 2001. 395p.

[5] Department of Health. The Expert Patient - A New Approach to Chronic Disease Management for the 21 st Century. London: Department of Health; 2001.

[6] Riordain RN, Meaney S, McCreary C. Impact of chronic oral mucosal disease on daily life: preliminary observations from a qualitative study. Oral Dis 2011; 17(3):265-9. https://doi.org/10.1111/j.1601-0825.2010.01734.x

[7] Streiner DL, Norman GR. Health Measurement Scales: A Practical Guide to Their Development and Use. Oxford: Oxford University Press; 2006.

[8] Travess HC, Newton JT, Sandy JR, Williams AC. The development of a patient-centered measure of the process and outcome of combined orthodontic and orthognathic treatment. J Orthod 2004; 31(3):220-34. https://doi.org/10.1179/146531204225022434

[9] Riordain RN, McCreary C. Oral cancer - current knowledge, practices and implications for training among an Irish general medical practitioner cohort. Oral Oncol 2009; 45(11):958-62. https://doi.org/10.1016/j.oraloncology.2009.04.008

[10] Tabolli S, Bergamo F, Alessandroni L, Di Pietro C, Sampogna F, Abeni D. Quality of life and psychological problems of patients with oral mucosal disease in dermatological practice. Dermatology 2009; 218(4):314-20. https://doi.org/10.1159/000196973

[11] Paradisi A, Sampogna F, Di Pietro C, Cianchini G, Didona B, Ferri R, et al. Quality of life assessment in patients with pemphigus using a minimum set of evaluation tools. J Am Acad Dermatol 2009; 60(2):261-9. https://doi.org/10.1016/j.jaad.2008.09.014

[12] Mumcu G, Hayran O, Ozalp DO, Inanc N, Yavuz S, Ergun T, et al. The assessment of oral health-related quality of life by factor analysis in patients with Behcet's disease and recurrent aphthous stomatitis. J Oral Pathol Med 2007; 36(3):147-52. https://doi.org/10.1111/j.1600-0714.2007.00514.x

[13] Hegarty AM, McGrath C, Hodgson TA, Porter SR. Patient-centred outcome measures in oral medicine: are they valid and reliable? Int J Oral Maxillofac Surg 2002; 31(6):670-4. https://doi.org/10.1054/ijom.2002.0260

[14] Einarson S, Gerdin EW, Hugoson A. Oral health-related quality of life and its relationship to self-reported oral discomfort and clinical status. Swed Dent J 2014;38(4):169-78.

[15] Karbach J, Al-Nawas B, Moergel M, Daubländer M. Oral health-related quality of life of patients with oral lichen planus, oral leukoplakia, or oral squamous cell carcinoma. Oral Maxillofac Surg 2014; 72(8):1517-22. https://doi.org/10.1016/j.joms.2014.04.008

[16] Robbeertz AA, Lauf Jr RC, Rupp RL, Alexander DC. A qualitative assessment of dental care access and utilization among the older adult population in the United States. Gen Dent 2006; 54(5):361-5.

[17] Department of Health. Reforming Emergency Care - Practical Steps. London: Department of Health; 2001.

[18] Anderson R. Patient expectations of emergency dental services: a qualitative interview study. Br Den J 2004; 197(6):331-4. https://doi.org/10.1038/sj.bdj.4811652

[19] Anderson R, Thomas DW, Phillips CJ. The effectiveness of out-of-hours dental services: II. patient satisfaction. Br Den J 2005; 198(3):151-6. https://doi.org/10.1038/sj.bdj.4812046

[20] Czerninski R, Zadik Y, Kartin-Gabbay T, Zini A, Touger-Decker R. Dietary alterations in patients with oral vesiculoulcerative diseases. Oral Surg Oral Med Oral Pathol Oral Radiol 2014; 117(3):319-23. https://doi.org/10.1016/j.0ooo.2013.08.006

[21] Jovanovic A, Schulten EA, van der waal I. Referral pattern of patients with oral mucosal lesions in the Netherlands. Community Dent Oral Epidemiol 1992; 20(2):94-6. https://doi.org/10.1111/j.1600-0528.1992.tbo0685.x

[22] Haberland CM, Allen CM, Beck FM. Referral patterns, lesion prevalence, and patient care parameters in a clinical oral pathology practice. Oral Surg Oral Med Oral Pathol Oral Radiol Endod 1999; 87(5):583-8. https://doi.org/10.1016/s1079-2 104(99)70138-1

[23] Navabi N, Chamani G, Zareei MR, Jalali P. Referral patterns and medical malpractice in patients with oral mucosal lesions before attending in oral medicine clinic of Kerman dental faculty. J Med Coun Iran 2009; 27(3):317-23.

[24] Macpherson LM, McCann MF, Gibson J, Binnie VI, Stephen KW. The role of primary healthcare professionals in oral cancer prevention and detection. Br Dent J 2003; 195(5):277-81. https://doi.org/10.1038/sj.bdj.4810481

[25] Carter LM, Ogden GR. Oral cancer awareness of general medical and general dental practitioners. Br Dent J 2007; 203(5):E10. https://doi.org/10.1038/bdj.2007.630 
[26] Miller CS, Epstein JB, Hall EH, Sirois D. Changing oral care needs in the United States: the continuing need for oral medicine. Oral Surg Oral Med Oral Pathol Oral Radiol Endod 2001; 91(1):34-44. https://doi.org/10.1067/moe.2001.110439

[27] Haberland CM, Allen CM, Beck FM. Referral patterns, lesion prevalence, and patient care parameters in a clinical oral pathology practice. Oral Surg Oral Med Oral Pathol Oral Radiol Endod 1999; 87(5):583-8. https://doi.org/10.1016/s1079-2 104(99)70138-1

[28] Hashemipour MA, Aghababaie M, Mirshekari TR, Asadi-Shekaari M, Tahmasbi-Arashlow M, Tahmasbi-Arashlow F, et al. Exfoliative cytology of oral mucosa among smokers, opium addicts and non-smokers: a cytomorphometric study. Arch Iran Med 2013; 16(12):725-30. 